\title{
Temperature dependence of wavelength-averaged DGD on different buried fibers
}

David L. Harris ${ }^{1}$, Pradeep K. Kondamuri ${ }^{2}$, James Pan $^{1}$ and Christopher Allen ${ }^{2}$

(1) Sprint Corp. ATL. Burlingame CA 94010

(2) Lightwave Communication lab, University of Kansas, Lawrence KS 66046

\begin{abstract}
From our PMD measurements on three buried standard single-mode fibers, we observed strong correlation between wavelength-averaged DGD and ambient temperature without any time lag between them. However, the nature of correlation was different on the three fibers.
\end{abstract}

\section{Introduction}

Because the impairments caused by polarization mode dispersion (PMD) change stochastically with wavelength and time and have a quadratic dependence on bit rate, PMD is recognized as the major distance limitation factor for high bit rate transmission systems. Different studies showed variations concerning the speed of PMD in installed fibers from milliseconds to days [1, 2]. The consensus is that buried fibers should be more stable compared to aerial fiber. However, there have been other reports claiming that fibers buried alongside railroads can experience rapid variation. A recent report [3] showed unexpected monotonic functional wavelength dependence with temperature. It was reported that depending on the wavelength, the deviation in differential group delay (DGD) was positive, negative or neutral as a function of temperature. Because of the importance of mean DGD or wavelength-averaged DGD $\left.(<\text { DGD }\rangle_{\lambda}\right)$ in calculating system outage probabilities, we report on the correlation between changes in ambient temperature and $\langle\mathrm{DGD}\rangle_{\lambda}$.

In this paper, we report on the correlation between the $\langle\mathrm{DGD}\rangle_{\lambda}$ and ambient temperature of different buried fibers. The fiber spans we measured were buried alongside the railroad except for several manhole access points and a single bridge attachment. Inside these manholes, it is typical to have several tens of meters of slack fiber available for maintenance thus making the fiber susceptible to environmental changes. These exposed sections can act as a fluctuating polarization controller, where the total PMD vector of a fiber consisting of $m$ sections, each with a known rotation matrix $R_{n}$ that can be expressed using the concatenation

rule as $\quad \vec{\tau}=\sum_{n=1}^{m} R(m, n+1) \vec{\tau}_{n}$

\section{Measurement setup}

A commercial femtosecond PMD test unit was used to measure DGD on three different buried fibers (1, 2 and 3) under test (FUT). The test unit utilized a fully polarimetric interferometry (FPI) technique, using a broadband source with wavelengths between $1530 \mathrm{~nm}$ to $1600 \mathrm{~nm}$. The FPI technique uses Poincaré sphere analysis - mathematically equivalent to Jones Matrix eigenanalysis. Because both the source and polarimeter were inside the same box, the FUT was required to have a loop-back configuration. Fibers 1, 2 and 3 were all standard single mode fibers with lengths of $66 \mathrm{~km}(33 \mathrm{~km} \times 2), 104 \mathrm{~km}(52 \mathrm{~km} \mathrm{x} \mathrm{2)}$ and $66 \mathrm{~km}(33 \mathrm{~km} \times 2)$ respectively. Fiber 2 was on a separate route from fibers 1 and 3 . The measurements were repeated every two minutes with a total of about 2300 wavelength data points per scan.

\section{Results}

To show the PMD fluctuations on installed fibers, we plotted the $\langle\text { DGD }\rangle_{\lambda}$ as a function of time for each of the three fibers. On each plot, the hourly temperature was also recorded. Fig.1a shows the measured $\langle\text { DGD }\rangle_{\lambda}$ on fiber 1 for about 4 days as well as the hourly ambient air temperature recorded at one location as functions of time. The measurement started shortly after 6:00pm day 1 and ended shortly before 6:00pm day 4 . The $\langle\text { DGD }\rangle_{\lambda}$ repeatedly peaked and remained almost unchanged between 12:30am and 8:00am, then decreased to its lowest $\langle\text { DGD }\rangle_{\lambda}$ over $4 \frac{1}{2}$ hours. The lowest $\left\langle\right.$ DGD $>_{\lambda}$ also remained stable between 12:30pm and 8pm. Fig. 1a shows a surprising one-to-one relationship between the $\langle\mathrm{DGD}\rangle_{\lambda}$ fluctuation and ambient temperature. At night when the temperature is at its lowest, the $\langle\mathrm{DGD}\rangle_{\lambda}$ peaks and during the high day time temperature the $\langle\text { DGD }\rangle_{\lambda}$ falls to its lowest. We defined a new coefficient that should be important to the design of PMD compensators as the rate of change of $\langle\mathrm{DGD}\rangle_{\lambda}$ per change in temperature $\left(\mathrm{ps} / \mathrm{hr} /{ }^{0} \mathrm{C}\right)$. For fiber 1 , we measured the worst case (falling edge) and found the coefficient was about $1.75 \times 10^{-2} \mathrm{ps} / \mathrm{hr} /{ }^{0} \mathrm{C}$. 


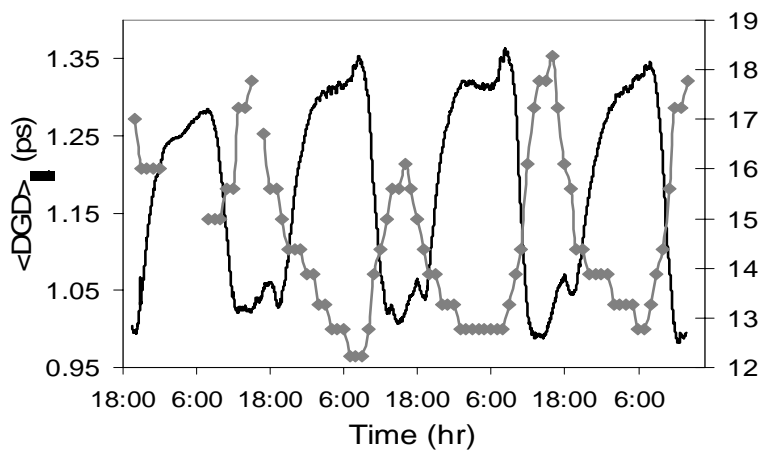

(a)

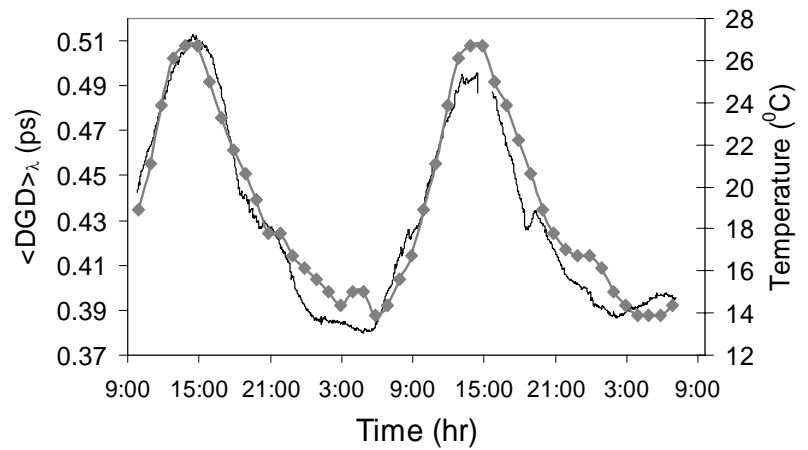

(b)

Fig. 1 Wavelength-averaged DGD and temperature (diamonds) as functions of time for (a) fiber 1, (b) fiber 2

Surprised by the inverse or negative correlation between $\langle\mathrm{DGD}\rangle_{\lambda}$ and temperature, we repeated the measurement on fiber 2 for two days. Fig. 1b shows a completely opposite correlation between the ambient temperatures and $\langle\mathrm{DGD}\rangle_{\lambda}$ compared to fig. 1 a. Notice how the change in temperature follows very well over time with the $\left\langle\right.$ DGD $>_{\lambda}$ evolution. We believe that the net rotation matrix which effectively orients the retarder at a random angle to be offset by $180^{\circ}$ in stokes space between fiber 1 and fiber 2 . For fiber 2 , we measured the worst case (falling edge) and found the coefficient was about $1.26 \times 10^{-3}\left(\mathrm{ps} / \mathrm{hr} /{ }^{0} \mathrm{C}\right)$.

Fig. 2 shows yet another different relationship between ambient temperature and $\langle\mathrm{DGD}\rangle_{\lambda}$ on fiber 3 . Unlike fig.1 ( $\mathrm{a}$ - b) where the change in temperature is either aligned positively of negatively with the DGD evolution throughout the test, fig. 3 shows both a negative and positive correlation over time. When the temperature goes above $\sim 62^{0} \mathrm{C}$ (day time), we observe positive correction. On the other hand, when the temperature falls below $62^{0} \mathrm{C}$ (night time), we observe negative correlation. This behavior was repetitive during the 3 days of testing. Because most of the fiber was buried and we observed no time lag in responses to the change in temperature, we can only contribute this behavior to the few sections that were exposed to temperature. The rate of change of $\langle\text { DGD }\rangle_{\lambda}$ per temperature change for fiber 3 was about $2 \times 10^{-3}\left(\mathrm{ps} / \mathrm{hr}{ }^{0} \mathrm{C}\right)$.

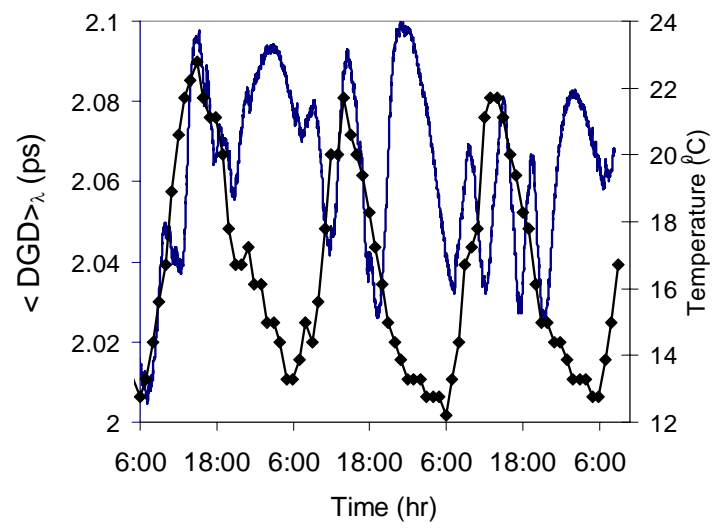

Fig. 2 Wavelength-averaged DGD and temperature (diamonds) as functions of time for fiber 3

\section{Conclusion}

We observed strong correlation between wavelength-averaged DGD and temperature on the three individual buried fibers. But, the nature of correlation was different on the three fibers. However, in all cases there was no time lag between the temperature variation and wavelength-averaged DGD variation. We attribute such strong correlation to the fact that a few sections of the buried fibers were exposed to temperature variations.

\section{References}

1. M. Brodsky et al., Photonics Technology Letters, 16(1), pp. 209-211, 2004.

2. C. Allen et al., Journal of lightwave technology, 21(1), pp. 79-86, 2003.

3. H. Bulow et al., Optical Fiber Communication Conference, Vol. 2, pp 83-85, 1999. 